\title{
V488 Per Revisited: No Strong Mid-infrared Emission Features and No Evidence for Stellar/substellar Companions
}

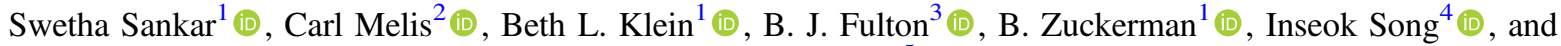 \\ Andrew W. Howard ${ }^{5}$ (iD \\ ${ }^{1}$ Department of Physics and Astronomy, University of California, Los Angeles, CA 90095-1562, USA; swethasnkr@ucla.edu \\ ${ }^{2}$ Center for Astrophysics and Space Sciences, University of California, San Diego, CA 92093-0424, USA; cmelis@ucsd.edu \\ ${ }^{3}$ NASA Exoplanet Science Institute/Caltech-IPAC, Pasadena, CA 91125, USA \\ ${ }^{4}$ Department of Physics and Astronomy, University of Georgia, Athens, GA 30602-2451, USA \\ ${ }^{5}$ California Institute of Technology, Pasadena, CA 91125, USA \\ Received 2021 May 17; revised 2021 July 28; accepted 2021 July 29; published 2021 November 23
}

\begin{abstract}
We present characterization of the planetary system architecture for V488 Per, the dustiest main-sequence star known with a fractional infrared luminosity of $\approx 16 \%$. Far-infrared imaging photometry confirms the existence of an outer planetary system dust population with a blackbody-fit temperature of $\approx 130 \mathrm{~K}$. Mid-infrared spectroscopy probing the previously identified $\approx 800 \mathrm{~K}$ inner planetary system dust population does not detect any obvious solidstate emission features, suggesting either large grain sizes that mute such emission and/or grain compositions dominated by species like amorphous carbon and metallic iron, which do not produce such features. In the latter case, the presence of significant quantities of iron-rich material could be indicative of the active formation of a Mercury-like planet around V488 Per. In any event, the absence of solid-state emission features is very unusual among main-sequence stars with copious amounts of warm orbiting dust particles; we know of no other such star whose mid-infrared spectrum lacks such features. Combined radial velocity monitoring and adaptive optics imaging find no evidence for stellar/substellar companions within several hundred astronomical units of V488 Per.
\end{abstract}

Unified Astronomy Thesaurus concepts: Circumstellar disks (235); Exoplanet systems (484); Variable stars (1761)

\section{Introduction}

Many main-sequence stars are now known to host substantial quantities of dusty material in their inner planetary systems (e.g., Melis 2016; Absil et al. 2021; Melis et al. 2021; Moór et al. 2021, and references therein). Where this inner planetary system dust comes from, what drives its evolution, and what its fate ultimately will be are matters of active research. Since this dust could be indicative of processes that might influence the formation, evolution, and habitability of Earth-like planets, it is essential to develop a firm understanding of it. Systematic investigation of the dust in these systems and their greater planetary system architecture will provide insight into the physical processes at play: e.g., how dust is generated and removed and the impact of dynamical perturbers on the origin and evolution of the dust.

Exceptionally dusty main-sequence stars are frequently found to reside in multiple stellar systems raising the question of whether or not multiplicity and chaotic dynamics contribute to generating these systems (e.g., Zuckerman 2015; Moór et al. 2021). For two older ( $>1$ Gyr) systems with mid-infrared excess emission indicative of inner planetary system dust, HD 69830 and $\mathrm{BD}+20^{\circ} 307$, it does indeed seem like additional planetary or stellar companions play a role in either destabilizing or corralling dust-producing rocky bodies leading to the unusually high levels of dust (e.g., Zuckerman et al. 2008; Payne et al. 2009).

Nesvold et al. (2016) study how a stellar-mass perturber orbiting exterior to and inclined to a planetesimal disk can excite the disk by the Kozai-Lidov mechanism resulting in dust-producing collisions. Moór et al. (2021) discuss how a widely orbiting stellar companion may act via Kozai-Lidov mechanisms to send outer planetary system bodies on inner planetary system-crossing orbits where they may release dust via disruption through sublimation or collisions.

Planetary mass companions orbiting a star may undergo a dynamical instability similar to the Late Heavy Bombardment hypothesized to have occurred in the solar system (e.g., Gomes et al. 2005). Bonsor et al. (2013, 2014) investigate such scenarios numerically, finding that sustained dust production in the inner planetary system can only be achieved when a planet migrates into a planetesimal belt. Fujiwara et al. (2009, 2012b) explore such a possibility in two stars with dusty inner planetary systems, rejecting it as an explanation for HD 15407A due to the lack of a detectable outer planetary system disk. However, Lisse et al. (2012) strongly suggest such a model is appropriate for $\eta \mathrm{Crv}$ based on a detected outer planetary system disk and possible water-ice features seen in its disk mid-infrared spectrum. Melis et al. (2021) invoke instabilities in tightly packed inner planetary systems of small planets (Earth to Neptune sizes) to produce the observed very dusty main-sequence stars.

Collisionally produced dust particles with sizes $\lesssim 1 \mu \mathrm{m}$ typically generate solid-state emission features in midinfrared spectra around wavelengths of $10 \mu \mathrm{m}$. Characterization of solid-state emission features can provide insight into where the dust in extreme debris disks originates from and what physical processes play a role in its evolution (e.g., Honda et al. 2004; Song et al. 2005; Chen et al. 2006; Lisse et al. 2007, 2008, 2009, 2012, 2017, 2020; Rhee et al. 2007, 2008; Currie et al. 2011; Johnson et al. 2012; Olofsson et al. 2012; Melis et al. 2013; Ballering et al. 2014; Morlok et al. 2014; Mittal et al. 2015). For example, highly energetic impacts that result in the production of crystalline silicates or silica (e.g., Lisse et al. 2009) would be suggestive of dynamically hot populations of mature rocky bodies pointing to the need for eccentricity excitation mechanisms. 


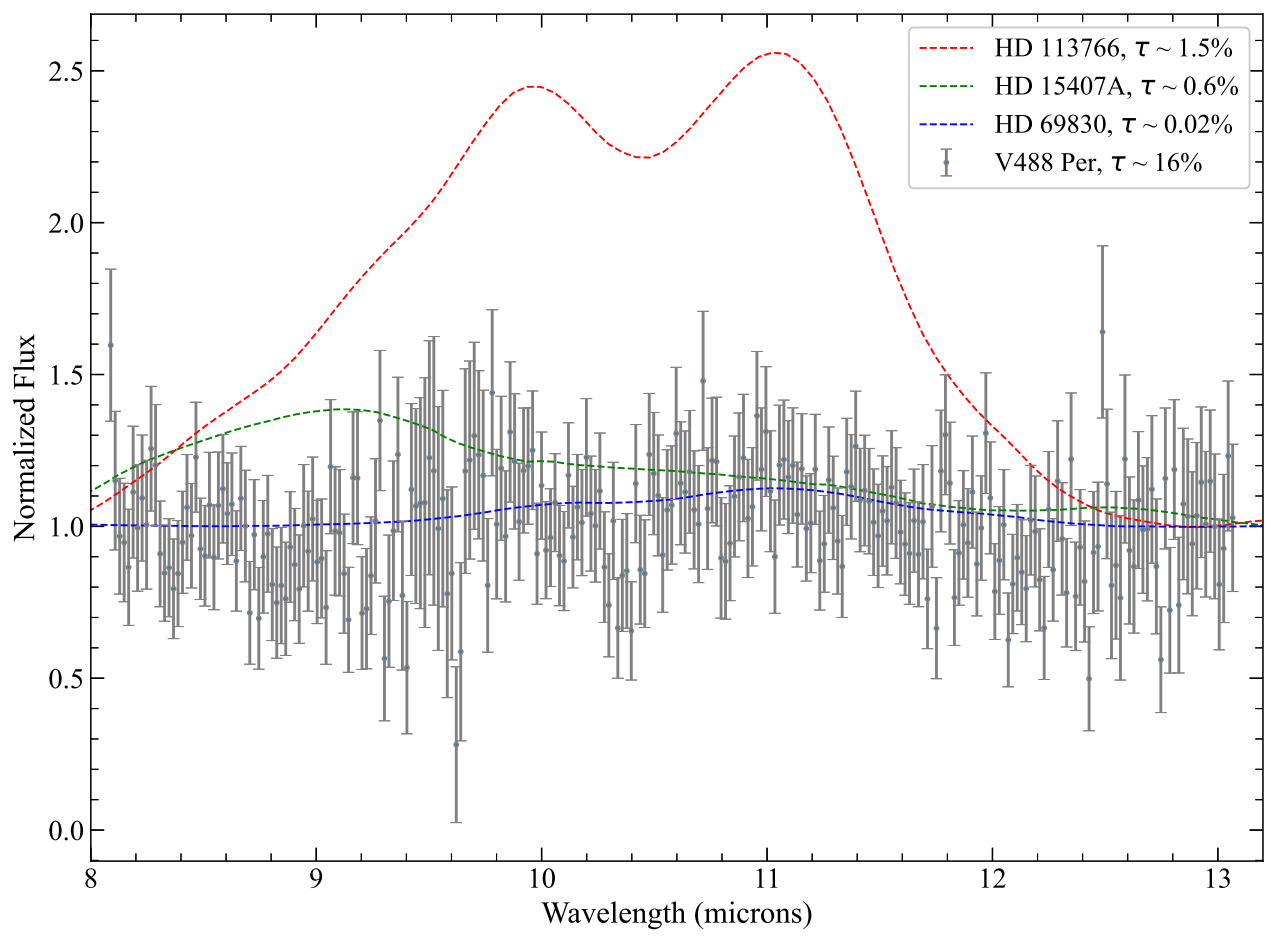

Figure 1. Mid-infrared spectra of V488 Per and exemplar main-sequence stars with warm inner planetary system dust. Each spectrum is normalized by a blackbody model fit to regions of the spectrum that do not have solid-state emission features, typically near 7.8 and $13.5 \mu \mathrm{m}$. The signal-to-noise ratio per pixel for the exemplar spectra ranges between roughly 100 and 200 and as such the error bars for those spectra are comparable to the thickness of the lines used to plot them. The fractional infrared luminosity ( $\left.\tau=L_{\mathrm{IR}} / L_{\mathrm{bol}}\right)$ of each source shown is given in the legend. HD 113766 (e.g., Chen et al. 2006; Lisse et al. 2008) is an exemplar for amorphous and crystalline solid-state emission features seen in stars with fractional infrared luminosities $\gtrsim 1 \%$; such sources present a range of solid-state emission feature peak strengths of $2-5 \times$ the continuum level. HD 15407A (e.g., Melis et al. 2010; Fujiwara et al. 2012a) is an exemplar for silica-dominated disks around main-sequence stars; such sources present solid-state emission feature peak strengths $\approx 1.5 \times$ the continuum level. HD 69830 (e.g., Beichman et al. 2005; Lisse et al. 2007) is an exemplar for the types of emission features seen around main-sequence stars with lower fractional infrared luminosities $(\sim 0.01 \%)$. Section 3.2 .2 provides further discussion.

Thus far, most extreme debris disk stars have mid-infrared spectral features from silicate species indicating large quantities of highly processed material (crystalline and silica grains) in addition to less processed amorphous species (e.g., Figure 1). However, it is worth noting three unusual systems that might be host to rather different dusty material (Melis et al. 2013; Lisse et al. 2017). Systems with atypical dust properties could provide information about unique dust stoichiometry and hence composition or dust production processes. Thorough characterization and modeling of mid-infrared spectral features for the dustiest main-sequence stars is essential in developing a complete picture for the types of compositions at a mineralogical level these systems host and the dynamical conditions that lead to their production through collisional processes (e.g., Lisse et al. 2009, 2012; Meng et al. 2014; Su et al. 2020).

We seek to obtain constraints on the overall planetary system architecture for the dustiest main-sequence stars known. V488 Per, a solar-type star belonging to the $\approx 80$ Myr old $\alpha$ Persei cluster (Soderblom et al. 2014), is of particular interest as it hosts what is currently the largest known fractional infrared luminosity for a main-sequence star of $\tau\left(=L_{\mathrm{IR}} / L_{\mathrm{bol}}\right) \approx 16 \%$ (Zuckerman et al. 2012). Basic stellar properties for V488 Per are given in Table 1. Zuckerman et al. (2012) suggest that V488 Per is possibly host to two separate belts of dust with temperatures of $\approx 820$ and $120 \mathrm{~K}$, but the basis for the cooler dust component was a single Wide-field Infrared Survey Explorer (WISE) excess measurement at $22 \mu \mathrm{m}$.

In this paper, we present new data that further characterize the dust and planetary system architecture of V488 Per.
Table 1

V488 Per Stellar Parameters

\begin{tabular}{lcl}
\hline \hline Parameter & Value & \multicolumn{1}{c}{ Reference } \\
\hline$V_{\text {mag }}$ & $12.89 \pm 0.05$ & Henden et al. (2015) \\
$\pi$ (mas) & $5.763 \pm 0.012$ & Gaia Collaboration et al. (2021) \\
$T_{\text {eff }}(\mathrm{K})$ & 4700 & This paper, Figure 4 \\
$R_{\star}\left(R_{\odot}\right)$ & 0.76 & This paper, Figure 4 \\
$L_{\star}\left(L_{\odot}\right)$ & 0.25 & This paper \\
$M_{\star}\left(M_{\odot}\right)$ & 0.84 & Wright et al. (2011); Stassun et al. (2019) \\
$i\left(^{\circ}\right)$ & $45 \pm 12$ & This paper, Section 3.1.1 \\
\hline
\end{tabular}

Section 2 details the new data sets, Section 3 discusses analyses based on measurements from the data, and Section 4 discusses possible interpretations and future observations to investigate them.

\section{Observations}

\subsection{Optical Spectroscopy}

Optical echelle spectra for V488 Per and a radial velocity standard of similar temperature class, HR 124 (Nidever et al. 2002), were collected at the Lick Observatory with the Automated Planet Finder (APF) telescope and Levy spectrograph (Vogt et al. 2014), as well as the Shane telescope and Hamilton spectrograph (Vogt 1987; Pakhomov \& Zhao 2013). We obtained these data to search for Doppler signatures from any companions to V488 Per at separations that could not be probed with imaging techniques; the iodine cell was not used due to the optical 
Table 2

Lick Observatory Radial Velocity Measurements

\begin{tabular}{lcllc}
\hline \hline UT Date & HJD & Instrument & SNR $^{\mathrm{a}}$ & $\begin{array}{c}V_{r} \\
\mathrm{~km} \mathrm{~s}^{-1}\end{array}$ \\
& & & & $-0.383 \pm 0.244$ \\
\hline 2016 Mar 01 & $2,457,448.75332$ & APF & 10.14 & $-0.433 \pm 0.160$ \\
2016 Nov 01 & $2,457,716.90075$ & APF & 18.03 & $-0.578 \pm 0.155$ \\
2017 Aug 21 & $2,457,987.00913$ & APF & 19.81 & $-0.53 \pm 0.158$ \\
2017 Sep 18 & $2,458,014.75714$ & APF & 21.09 & $-0.420 \pm 0.164$ \\
2017 Oct 04 & $2,458,030.81506$ & APF & 19.66 & $-0.530 \pm 0.158$ \\
2017 Oct 30 & $2,458,056.78022$ & APF & 21.20 & $-0.423 \pm 0.142$ \\
2017 Dec 08 & $2,458,095.88214$ & APF & 20.91 & $-0.490 \pm 0.142$ \\
2018 Feb 08 & $2,458,157.68050$ & APF & 19.31 & $-0.375 \pm 0.14$ \\
2018 Oct 17 & $2,458,408.78883$ & APF & 22.19 & $-0.436 \pm 0.113$ \\
2018 Dec 13 & $2,458,465.72084$ & APF & 11.68 & $-0.410 \pm 0.257$ \\
2020 Aug 07 & $2,459,068.99811$ & Hamilton & 18.60 & $-0.409 \pm 0.244$ \\
\hline
\end{tabular}

Note.

${ }^{a}$ The signal-to-noise ratio per pixel, measured near $6475 \AA$.

faintness of V488 Per for these telescopes (Table 1). Twodimensional raw frames are bias subtracted, flat-fielded, extracted into one-dimensional spectra through straight summing of flux inside an aperture defined for each individual echelle order, and finally wavelength calibrated with comparison lamp spectra. Radial velocities are measured through cross correlation with HR 124 and corrected to the heliocentric reference frame. Table 2 reports observation epochs, data quality, and measured velocities, while Figure 2 presents measurements and associated analysis.

\subsection{TESS}

To help assess the stellar inclination angle and hence better interpret Doppler mass limits by removing the $\sin i$ ambiguity, we downloaded and analyzed Transiting Exoplanet Survey Satellite (TESS) light-curve data for V488 Per and other $\alpha$ Per stars with similar spectral type and spectroscopic projected rotational velocities (Stauffer et al. 1985). All light curves are generated from Full Frame Imaging data acquired in Sector 18 from 2019 November 03-2019 December 16. Data products are MIT Quick Look Pipeline (QLP; Huang et al. 2020a, 2020b) light curves as downloaded from the Mikulski Archive for Space Telescopes (MAST). Some times with bad data are removed by hand before analysis. Figure 3 shows the TESS light curves used for this work.

\section{3. $A O$}

Diffraction-limited thermal-infrared adaptive optics (AO) imaging of V488 Per was obtained at the Keck Observatory with the facility AO system (Wizinowich et al. 2000; Wizinowich 2013) and Near-Infrared Camera 2 (NIRC2) camera on UT 2013 Feb 03. We conducted observations using the $L^{\prime}$ filter (central wavelength and bandpass of $3.776 \pm$ $0.700 \mu \mathrm{m}$ ) and the narrow camera setting, resulting in a plate scale of $0.009952^{\prime \prime}$ pixel $^{-1}$ (Yelda et al. 2010). We obtained these data to further rule out possible contamination of V488 Per by a background object and then to perform a shallow search for possible companion stars. The excess emission level for V488 Per at a wavelength of $\approx 3.8 \mu \mathrm{m}$ is just over twice the stellar photosphere emission level (Figure 4), thus any contaminating object that would be responsible for the apparent infrared excess emission toward V488 Per would need to be of comparable brightness as V488 Per (the star) alone.
Seven dithered exposures of $3.0 \mathrm{~s}$ each $(0.3 \mathrm{~s}$ integration $\times 10$ coadds) were obtained resulting in a total integration time of $21 \mathrm{~s}$. Background-subtracted and flat-fielded individual images are registered, then combined to produce a final image for analysis, which is displayed in Figure 2.

\subsection{COMICS Spectra}

We collected Cool Mid-Infrared Camera and Spectrometer (COMICS; Kataza et al. 2000; Okamoto et al. 2003) $R \approx 170$ mid-infrared spectroscopic data from the Subaru $8.2 \mathrm{~m}$ Telescope on UT 2014 November 06. The night featured seeing in the mid-infrared of $\approx 0.4^{\prime \prime}$ and a precipitable water vapor value of $\sim 4 \mathrm{~mm}$. Observations and data reduction follow those of Su et al. (2020), and important aspects of observations of V488 Per are discussed here.

The observing strategy for V488 Per started with observations of the calibrator star HD 21552 (K3 III), a sequence on V488 Per lasting $\approx 90$ minutes of wall-clock time, a second visit to HD 21552 followed by another $\approx 90$ minute sequence of observations on V488 Per, then a final visit to HD 21552. All exposures for both stars were chopped in an ABAB pattern keeping all beams on slit. V488 Per is faint for ground-based mid-infrared astronomy (Figure 4), so each saveset obtained for it had 222 total exposures or 111 chop pairs, the accumulation of which was sufficient to see the spectral signal. By comparison, the bright calibrator only needed 24 total exposures or 12 chop pairs to obtain high $(\sim 50)$ signal-tonoise. During reduction, each chop pair is differenced and examined for quality. Those chop pairs showing strong residual structure across the detector (indicating rapidly varying background conditions) are discarded. Chop pairs that are kept are then rectified along the spatial axis such that night sky emission lines run vertically only (initially they are slightly tilted horizontally). A second pass of background removal is then done by subtracting at each dispersion pixel location the median value of background-only pixels along the spatial axis. At the end of the assessment, roughly two-thirds of the first spectral sequence on V488 Per was kept and roughly one-third of the second spectral sequence.

Fully processed chop pairs in each sequence are mediancombined and the positive and negative spectral beams are extracted via a straight aperture sum. Uncertainties on spectral samples are calculated by determining the rms of backgroundonly pixels and summing that in quadrature for the number of pixels in the aperture and the Poisson noise on the total summed flux in the aperture. This is done for each of the two target spectral sequences and for spectra from each visit to the calibration star.

For each visit to the calibrator we scaled and combined positive and negative beam spectra with a weighted mean, then used one spectrum from the set of three visits to correct the telluric absorption in each positive and negative spectrum for V488 Per. Telluric correction is done by dividing a science spectrum by one of the calibrator visit spectra shifted in wavelength to provide the best cancellation of the strong $\sim 9.5 \mu \mathrm{m}$ ozone feature. For each science spectrum, we selected the calibrator spectrum that resulted in the lowest rms residuals after telluric feature removal; in practice, this was the calibrator spectrum obtained in the middle of the two sequences on V488 Per.

Corrected science target positive and negative beam spectra in each sequence were then scaled and combined via weighted 

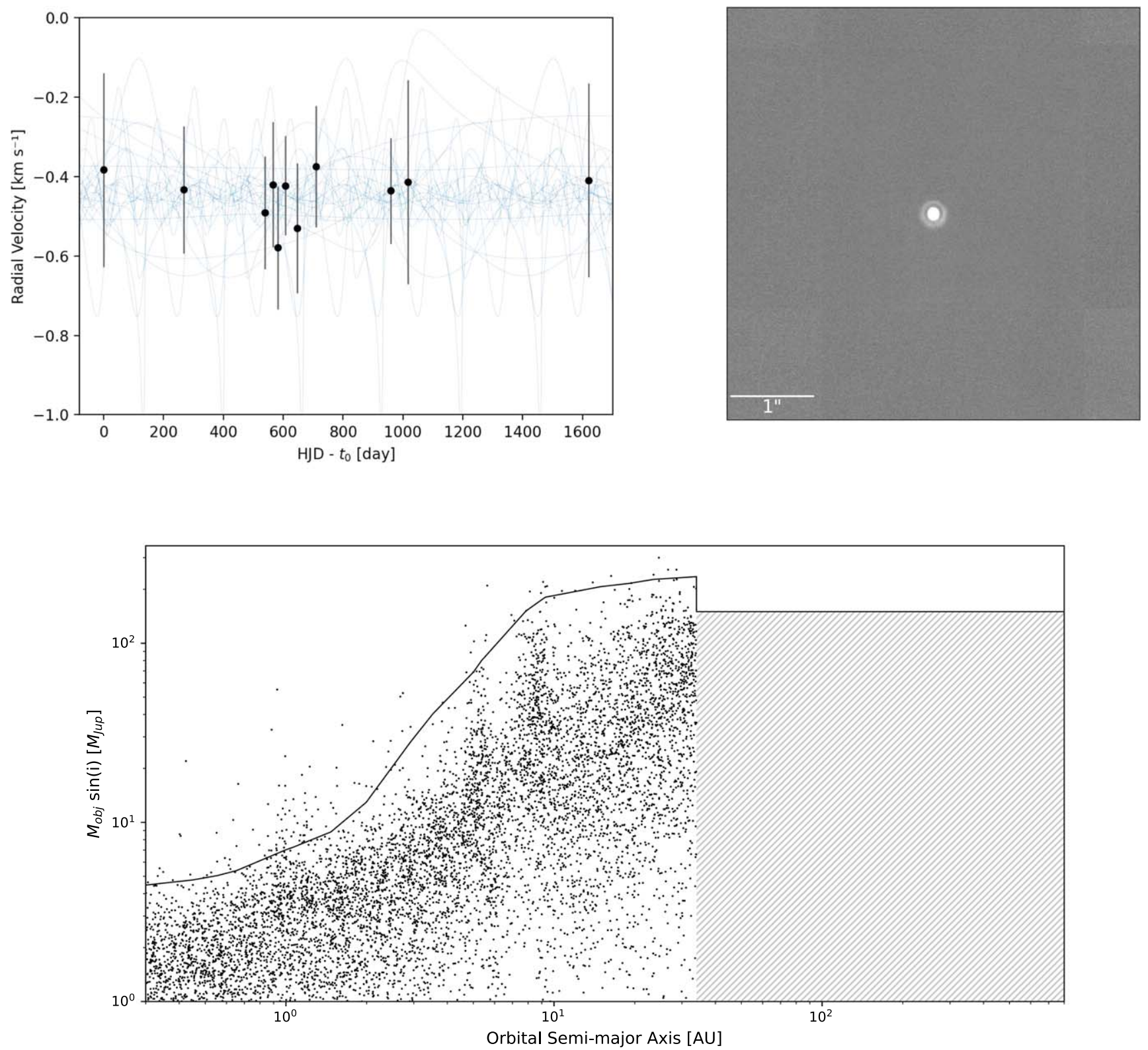

Figure 2. Radial velocity monitoring and adaptive optics (AO) imaging results and mass sensitivity limits derived from them. Top left panel: time series of radial velocity measurements for V488 Per (black dots with error bars) with a selection of representative The Joker allowable solutions overplotted (blue dotted curves). $t_{0}$ is HJD 2,457,448.75332 (UT 2016 March 01), the date of the first observation of V488 Per at the Lick Observatory. The median radial velocity for all measurements shown is $-0.423 \mathrm{~km} \mathrm{~s}^{-1}$. Top right panel: Keck AO L' image of V488 Per; the first airy ring is clearly visible around the star. North is up and East is left, no other sources are detected in the imaged field of view. Bottom panel: mass sensitivity limits obtained as described in Section 3; masses are $m$ sini only for the spectroscopic curve covering semimajor axes $<30$ au, there is no inclination angle ambiguity for the AO imaging mass sensitivity line shown for semimajor axes $>30$ au. Dots are from allowable solutions obtained with The Joker, the hatched region shows companions that would not have been detected with AO imaging; above the solid curve we expect we would have detected any such companions if they were present.

mean. A wavelength scale is determined based on a low-order polynomial fit to the position of known bright sky emission lines. A Rayleigh-Jeans slope is used as an approximation for the calibrator spectral shape and applied to the corrected science target spectra to arrive at relative flux-calibrated spectra (i.e., the spectral shape is robust, but the absolute flux level is not).

To obtain an accurate absolute flux scaling for the spectra for each sequence we measured photometry from images of the target and calibrator. A short imaging acquisition sequence with the N12.4 filter (bandpass of $12.4 \pm 1.2 \mu \mathrm{m}$ ) was obtained for V488 Per and the calibrator star HD 21552 in chopping-only mode. Chop pairs were differenced to remove background emission and aperture photometry performed on the science target and calibrator star. We note that only a single point source was seen in the V488 Per field. With an adopted flux density for HD 21552 of $9.11 \mathrm{Jy}$ (extrapolated from the WISE-measured $11.56 \mu \mathrm{m}$ flux density of $10.48 \pm 0.11 \mathrm{Jy}$ ), we measure a $12.4 \mu \mathrm{m}$ flux density for V488 Per of $60 \pm 16 \mathrm{mJy}$. Since this is consistent within the errors with the WISE $11.56 \mu \mathrm{m}$ flux measurement for V488 Per of $41.0 \pm 0.6 \mathrm{mJy}$, we adopt the WISE value to set the spectrum absolute flux level. These absolute flux scales are accurate at the $\approx 10 \%$ level (this error source is not included in the spectral uncertainties). 

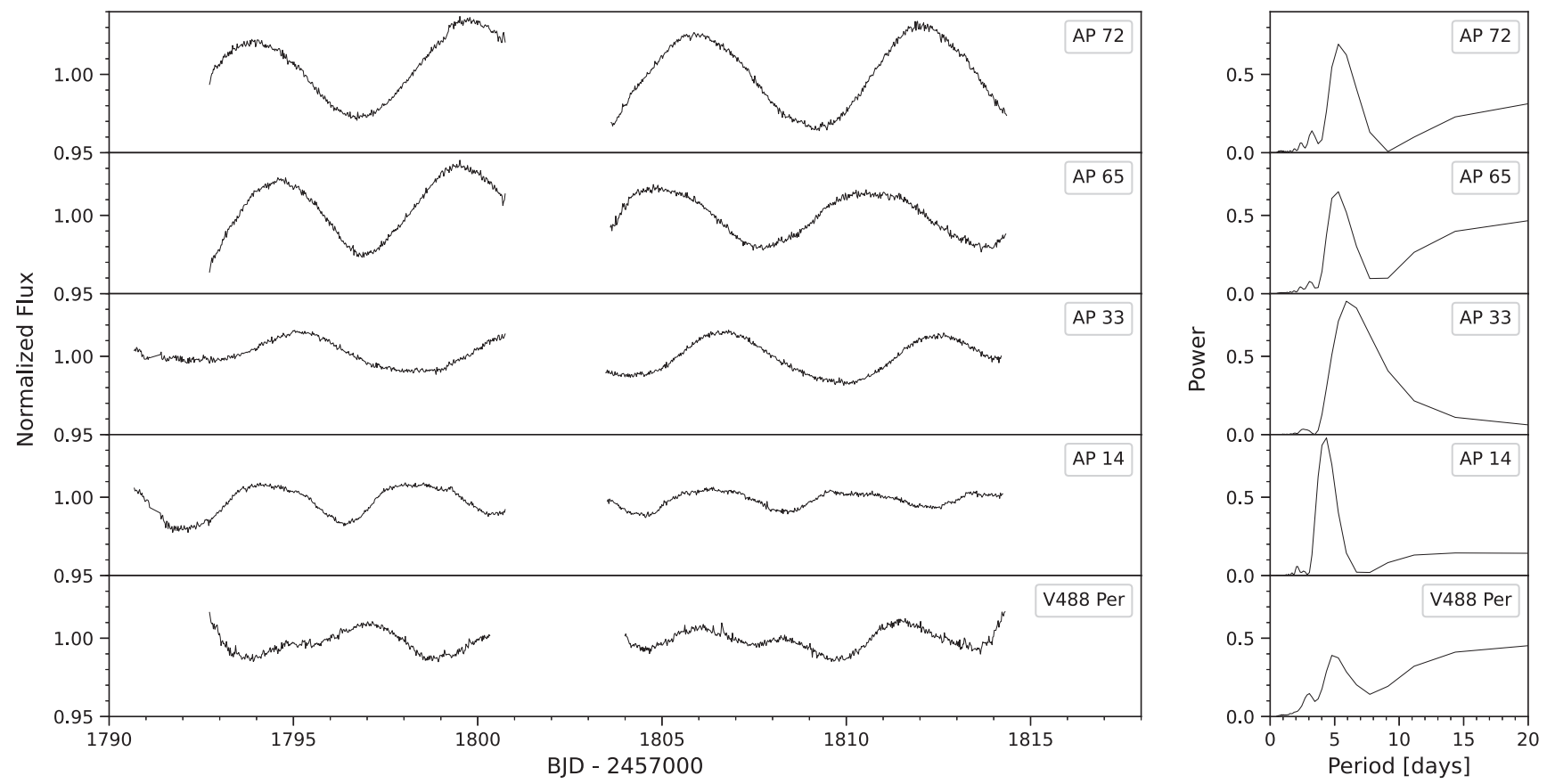

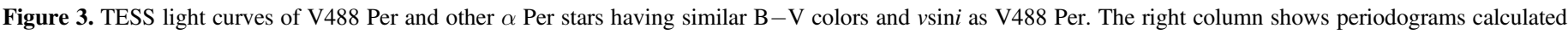

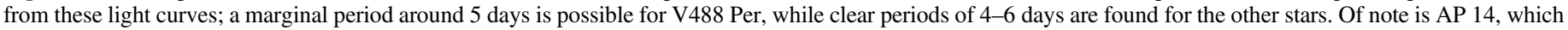
shows a reduction in the amplitude of its periodic signal over the TESS monitoring period and ends with variability levels comparable to V488 Per.

A final $N$-band spectrum for V488 Per is then obtained by averaging the two separate sequences together. The final spectrum signal-to-noise level is $\approx 6$ near $11.5 \mu \mathrm{m}$.

\subsection{Herschel Imaging}

Simultaneous far-infrared observations at 70 and $160 \mu \mathrm{m}$ were obtained with the ESA Herschel Space Observatory (Pilbratt et al. 2010) using the PACS imaging photometer (Poglitsch et al. 2010). V488 Per was observed on UT 2012 September 11 (Proposal OT2_cmelis_3; OBSIDs 1342250847 and 1342250848) in Mini Scan map mode. Scan legs of $3^{\prime}$ length were observed at medium speed with two orientation angles of $70^{\circ}$ and $110^{\circ}$ in the array coordinates. High level image products are obtained from the Herschel Science Center and aperture photometry is performed on these images as described in Vican et al. (2016). We obtain a $70 \mu \mathrm{m}$ flux density in agreement with the Herschel PACS Point Source Catalogue (Herschel Point Source Catalogue Working Group et al. 2020) and adopt their value; we report for V488 Per a $70 \mu \mathrm{m}$ flux density of $66.5 \pm 6.5 \mathrm{mJy}$ and $160 \mu \mathrm{m}$ flux density of $22 \pm 10 \mathrm{mJy}$.

\section{Analysis and Discussion}

\subsection{Search for Companions}

Figure 2 reveals the overall stability of the radial velocity measurements made at the Lick Observatory. A periodogram search does not reveal any signals hidden in the noise, and with no obvious signal present in the data we proceed to estimate mass sensitivity limits. Radial velocity measurements given in Table 2 are also consistent with values previously measured for V488 Per (Stauffer et al. 1985; Mermilliod et al. 2008; Zuckerman et al. 2012). The measurement from Mermilliod et al. (2008), taken on HJD 2,449,655.560 (UT 1994 October 30) and having a value of $-0.31 \pm 0.15 \mathrm{~km} \mathrm{~s}^{-1}$, is incorporated into our companion sensitivity analysis. The other two measurements are not utilized as they either did not have information regarding time of observation (Stauffer et al. 1985) or because of inaccuracies resulting from a much later spectral type radial velocity standard used for cross correlation (Zuckerman et al. 2012).

To estimate mass sensitivity limits as a function of orbital separation we utilized The Joker (Price-Whelan et al. 2017) and adopted a stellar mass of $0.84 M_{\odot}$ (Table 1$)$. The Joker is designed to characterize a two body system with the requirement that the system behave similarly to a single-lined spectroscopic binary and exhibit variability in radial velocity measurements. It takes the input of multi-epoch radial velocity data with errors and by Monte Carlo analysis produces a suite of possible fits for the data. We conducted a set of The Joker runs with a uniform prior distribution covering a variety of orbital period ranges; in all runs, priors on the velocity semiamplitude and the systemic velocity were taken to be Gaussians with $\sigma$ of 3 and $75 \mathrm{~km} \mathrm{~s}^{-1}$, respectively. Discrete orbital period range bins are constructed out to a maximum period of 60,000 days $\left(\approx 30\right.$ au for a circular orbit). For each run, $10^{3}$ priors are generated but only a subset survive the rejection sampling step of The Joker. Figure 2 presents some example orbital fits that are consistent with the available radial velocity data. Five runs per period range bin are executed and a mass sensitivity for that bin is calculated from the median of the maximum masses obtained from each of the five runs. The resulting mass-period sensitivity curve from these samplings is displayed in Figure 2; it is noted that mass sensitivities obtained in this way are $m \sin i$.

Radial velocity mass limits become increasingly insensitive with orbital period, especially beyond the maximum time baseline covered as shown in Figure 2. To supplement mass sensitivities at larger orbital separations we make use of the AO data. One other star was observed with the $L^{\prime}$ filter on the same night as V488 Per, but at a different time and significantly different airmass and as such point-spread-function (PSF) 


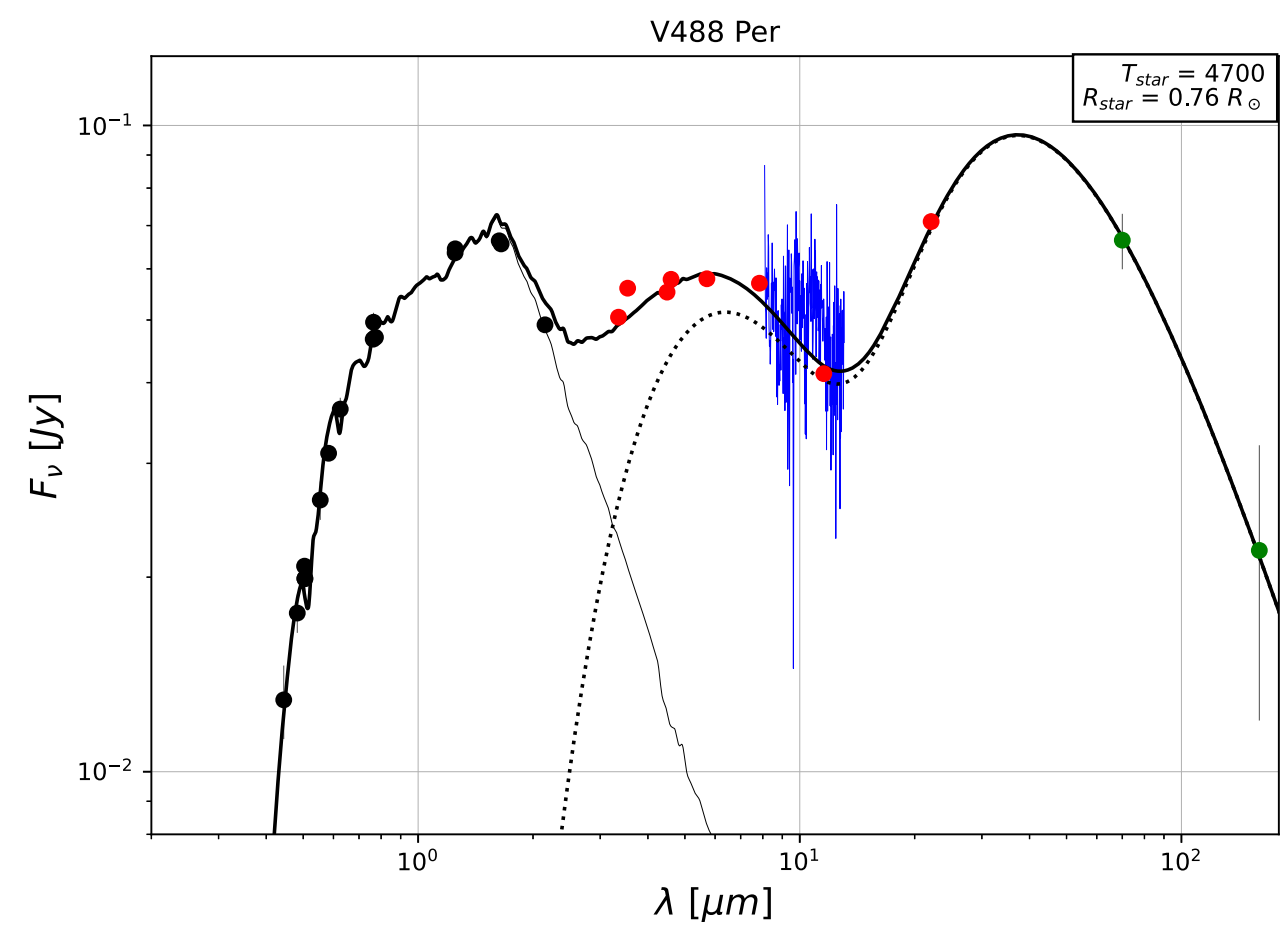

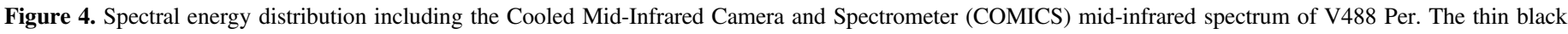

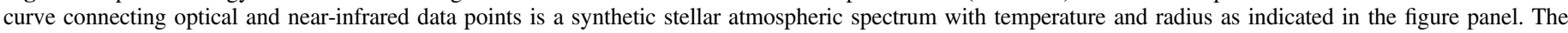

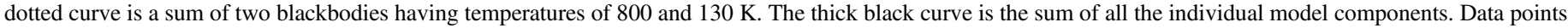

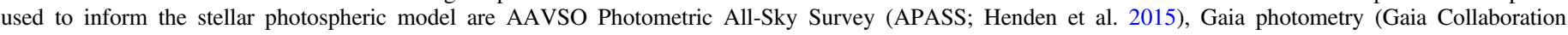

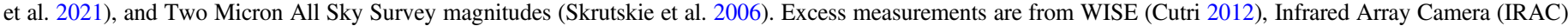

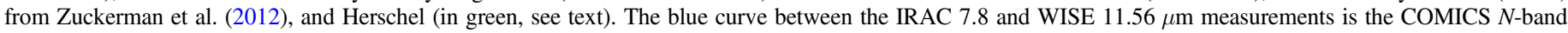
spectrum. Vertical lines in data points indicate the measurement uncertainty. Some measurement uncertainties are smaller than the point sizes on the plot.

subtraction was not fruitful. However, in general the PSF of V488 Per matched that of the other observed star indicating a single source. Figure 2 shows what we conclude is a single, diffraction-limited point source in the $L^{\prime}$ AO image.

There is no seeing-limited halo evident in the final image and thus the sensitivity beyond the first airy ring is determined only by the total amount of integration time covered at that position. The best sensitivity is obtained in an annulus with inner radius just outside the first airy ring at $0.19^{\prime \prime}$ to an outer radius of $4.72^{\prime \prime}$ from V488 Per. Adopting the Gaia EDR3 parallax (Table 1) results in a distance to V488 Per of $173.5 \pm 0.4 \mathrm{pc}$ and separation-space probed by the $L^{\prime}$ AO image of 33-820 au.

Interpolating between the WISE W1 and W2 magnitudes, we obtain an $L^{\prime}$ magnitude for V488 Per of $\approx 9$. Using V488 Per as a comparison star, we estimate that we are sensitive to point sources as faint as $L^{\prime} \sim 13$ magnitudes at the $5 \sigma$ level in the best sensitivity areas of the AO image. Consulting Leggett (1992) and Chabrier et al. (2000), and using the adopted age for the $\alpha$ Per cluster of $80 \mathrm{Myr}$ (see Section 1), we obtain mass limits of $\sim 0.15 M_{\odot}$; we show this mass limit in relation to the Doppler monitoring mass sensitivities in Figure 2. This mass limit is more sensitive than the $\sim 0.6 M_{\odot}$ limit obtained from Hubble Space Telescope $1.3 \mu \mathrm{m}$ imaging reported in Patience et al. (2002).

We do not assess the binarity of V488 Per beyond $800 \mathrm{au}$; Moór et al. (2021) discuss problems with the widely separated candidate companions identified in Zuckerman (2015).

\subsubsection{Stellar Inclination}

It is desirable to try and remove the $\sin i$ ambiguity in the Doppler mass sensitivity limits. Without any detected companions (nor resolved imaging of the circumstellar disks) it is not possible to assess the inclination angle of the planetary system around V488 Per. As a proxy, we will use the inclination angle of the stellar rotation axis, while noting that it need not be the case that the two axes are aligned.

With a measurement of the rotation period of V488 Per from photometric monitoring and the rotational broadening of the stellar photospheric absorption lines from APF data we can obtain the stellar inclination angle. From the UT 2017 December 08 APF spectrum we measure a $v \sin i$ value of $4.5 \pm 1.5 \mathrm{~km} \mathrm{~s}^{-1}$ following the methodology of Strassmeier et al. (1990). To obtain the stellar rotation period we analyzed TESS light-curve data. However, the TESS data reveal only mild variability for V488 Per, not the clear periodic spot modulation that would be expected for a young, Sun-like star (Figure 3).

The result from TESS stands in stark contrast to variability measurements for V488 Per (=AP 70) in the literature. Past ground-based observations covering multiple rotational periods clearly detect rotational modulation of starspots on the surface of V488 Per, finding rotational periods of $123.5 \mathrm{hr}$ (or 5.15 days) in Stauffer et al. (1985), $6.4 \pm 0.1$ days in Allain et al. (1996), and 5.98 days in Heinze et al. (2018) (ATO J052.0779+48.6632, ATLAS ObjID 166390520779906601).

To place V488 Per in context, we examined TESS lightcurve data for other $\alpha$ Per stars with measured rotational periods in Stauffer et al. (1985) and Allain et al. (1996) that had $\mathrm{B}-\mathrm{V}$ colors and $v \sin i$ comparable to V488 Per (we considered stars with $v \sin i \lesssim 10 \mathrm{~km} \mathrm{~s}^{-1}$ from Stauffer et al. 1985). A comparison of these stars' TESS light curves to that for V488 Per is shown in Figure 3. Each of the additional examined stars 
shows clear periodic variability with periods on the order of 4-6 days.

Why does V488 Per not exhibit similar behavior as other $\alpha$ Per stars in the TESS data? We believe the answer is starspot cycles, and the star AP 14 shown in Figure 3 demonstrates the evolution of spot modulation amplitude over the TESS monitoring period ( $\approx 27$ days). In this interpretation, we just happened to catch V488 Per with TESS at the minimum of its starspot cycle, which had a duration of at least $\sim 1$ month. AP 14 was captured during its decay phase, which probably also took on the order of at least $\sim 1$ month. The other stars shown in Figure 3 were seen during times of near-maximum activity.

Based on this interpretation, we adopt the average of literature-measured rotational periods for V488 Per of 5.8 days. To calculate the stellar inclination angle, we further adopt the stellar radius of $0.76 R_{\odot}$ from the spectral energy distribution fit in Figure 4. With these values we calculate a stellar inclination angle of $45^{\circ} \pm 12^{\circ}$. This suggests that the spectroscopic mass sensitivity values shown in Figure 2 should be increased by a factor of $\sim 1.4$ if the stellar and planetary system inclination angles are the same.

\subsection{Circumstellar Dust Properties}

While the AO image is not capable of characterizing the dust around V488 Per, it does effectively eliminate any possibility that the excess emission is due to a background contaminating object. Any such object would be as bright as V488 Per in the $L^{\prime}$ band (Figure 4) and would be obvious even at separations interior to the first airy ring. We conclude with high confidence that all infrared excess emission seen toward V488 Per is associated with the star.

\subsubsection{Dust Architecture}

Figure 4 collects optical to far-infrared photometric measurements for V488 Per and fits them with a simplistic model consisting of a stellar photosphere and two blackbody components. The Herschel data clearly confirms that there exists a cooler dust population in the outer planetary system of V488 Per that was hinted at previously from WISE $22.09 \mu \mathrm{m}$ photometry (Zuckerman et al. 2012). The blackbody temperature of this outer dust component is reasonably constrained to $\approx 130 \mathrm{~K}$, while the inner dust population is fit acceptably with a blackbody having temperature of $\approx 800 \mathrm{~K}$.

We interpret the two dust populations as separate rings of material within the V488 Per planetary system. This interpretation is motivated by past results indicating separate inner and outer planetary system debris disks for other multipleblackbody component systems (e.g., Backman et al. 2009; Morales et al. 2009; Su et al. 2009; Kennedy \& Wyatt 2014; Vican et al. 2016). For the luminosity of V488 Per (calculated with the spectral energy distribution fit temperature and radius in $L=4 \pi R^{2} \mathrm{~T}^{4}$; see Table 1) this would place blackbodyemitting grains having the above temperatures at orbital separations of $\sim 0.07$ and $\sim 2.7 \mathrm{au}$. The widths of these two putative disks are not well constrained with the available data and can be better characterized if the grain composition can be determined (see below) and/or if resolved imaging can be obtained.

\subsubsection{Grain Composition}

Typically, strong solid-state emission features are seen in the mid-infrared spectra of exceptionally dusty main-sequence stars (e.g., Honda et al. 2004; Chen et al. 2006; Lisse et al. 2008; Melis et al. 2010; Olofsson et al. 2012; Lisse et al. 2017; Melis et al. 2021). V488 Per is thus unusual in not showing any obvious features in its COMICS $N$-band spectrum (Figures 1 and 5). Figure 1 compares the COMICS mid-infrared spectrum of V488 Per with Spitzer IRS spectra of exemplar extreme debris disk systems having well-detected solid-state emission features. To date, stars having fractional infrared luminosities $\gtrsim 1 \%$ all have strong solid-state emission features present in their mid-infrared spectra that can be ruled out in the spectrum of V488 Per (several new extremely dusty main-sequence stars have yet to be observed with mid-infrared spectrometers; e.g., those stars from Gaidos et al. 2019; Tajiri et al. 2020, and Moór et al. 2021). This stands for all types of dust grain species currently known, whether they be silica-rich (e.g., Chen et al. 2006; Rhee et al. 2008; Melis et al. 2010; Fujiwara et al. 2012a), dominated by amorphous and/or crystalline silicates (e.g., Honda et al. 2004; Song et al. 2005; Chen et al. 2006; Rhee et al. 2007; Olofsson et al. 2012; Melis et al. 2021), or stars with yet-to-be-identified emission features (e.g., Melis et al. 2013; Lisse et al. 2017). When compared against less dusty stars (fractional infrared luminosities $\sim 0.01 \%$ ), it is possible that the noise in the COMICS spectrum of V488 Per could hide weaker solid-state emission features. This is evidenced by comparison to HD 69830 (e.g., Beichman et al. 2005) in Figure 1, and is similarly true for other such systems (e.g., Chen et al. 2006; Moór et al. 2009; Mittal et al. 2015). While it is possible that some weaker solid-state emission features could be hidden in the COMICS spectrum presented for V488 Per, it remains the case that it is unusual when compared against other stars having huge amounts of inner planetary system dust $(\tau \gtrsim 1 \%)$.

We discuss some possible explanations for why no strong solidstate emission feature is seen in the COMICS spectrum of V488 Per and elaborate on them further here and in Section 4. To generate the observed featureless mid-infrared spectrum, either (1) only a weak solid-state emission feature is present as discussed above; (2) dust grains are larger than $\sim 10 \mu \mathrm{m}$ in size, at which point solid-state emission features near a wavelength of $10 \mu \mathrm{m}$ become severely muted (e.g., Figure 5; see also Min et al. 2007 and Section 4.3 of Olofsson et al. 2012); and/or (3) the dust composition could be dominated by species that do not produce obvious mid-infrared solid-state emission features, like amorphous carbon or metallic iron. Possibility (2) seems highly unlikely given that the observed dust is almost certainly produced via collisions of rocky bodies, which should eventually be ground down to radiation blow-out sizes $(\lesssim 0.1 \mu \mathrm{m}$ for V488 Per; e.g., Artymowicz 1988) before being removed from the planetary system.

To explore possibility (1) in more detail, we constructed solidstate emission feature models for V488 Per that would be consistent with the observed COMICS spectrum. In these models, we utilize a blackbody component for the disk continuum emission and an emission feature component computed from the linear combination of dust absorption coefficients multiplied by a blackbody; the solid angle of each component is adjusted to find agreement with the COMICS spectrum. We use dust absorption coefficients from Min et al. (2007) for silicates and silica grain species. Since emission from amorphous carbon or metallic iron is indistinguishable from continuum emission in the $8-13 \mu \mathrm{m}$ wavelength region (e.g., 

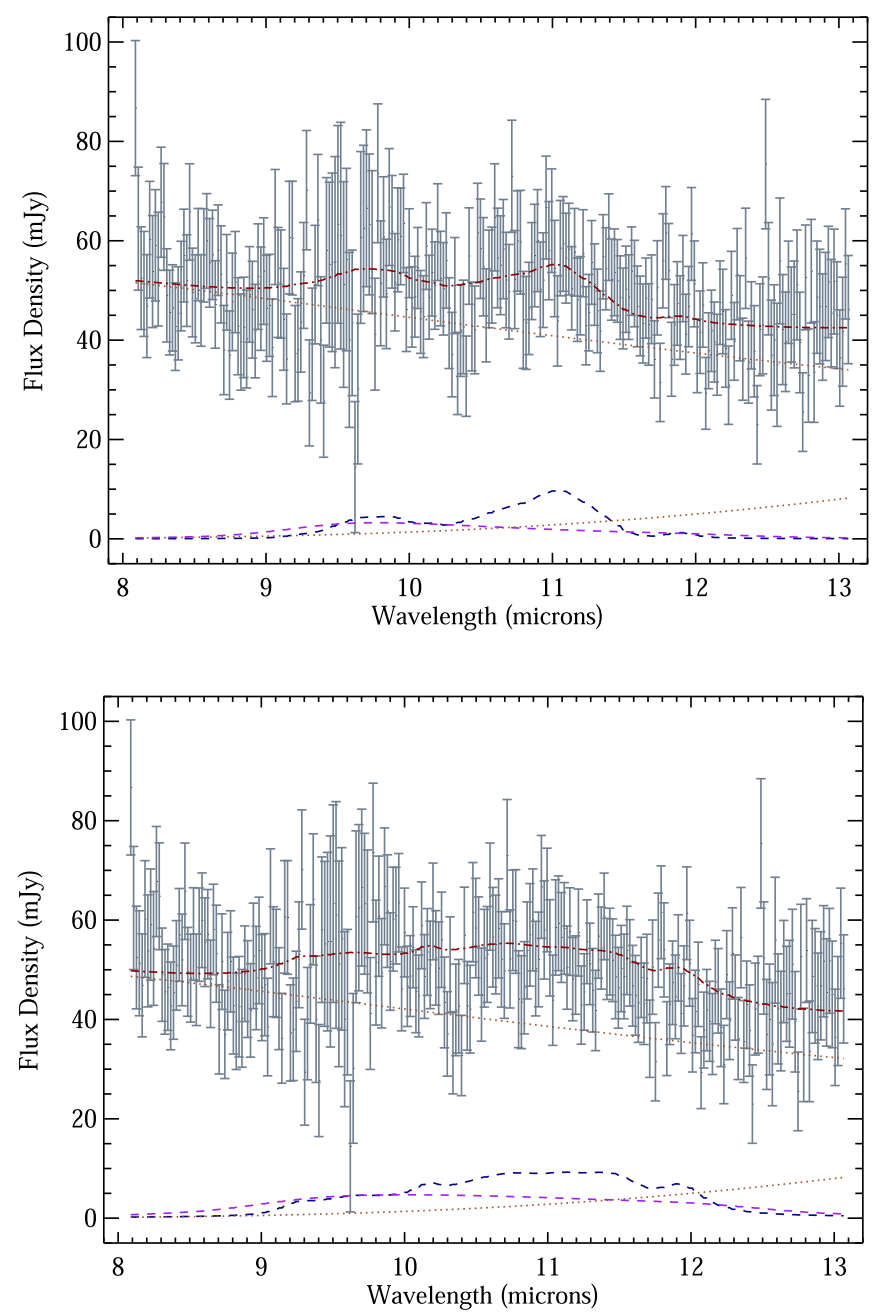

Figure 5. Example solid-state emission models that are consistent with the V488 Per COMICS spectrum. In each panel, the V488 Per spectrum is shown in gray (spectral samples and associated uncertainties), orange dotted curves are 800 and $130 \mathrm{~K}$ blackbodies, purple and blue dashed curves are amorphous and crystalline silicates, respectively, and the red dotted-dashed curve is the sum of all components shown. We do not include amorphous carbon or metallic iron in these models as their emission is indistinguishable from that of the continuum components; it is not possible to constrain amorphous carbon or metallic iron with the available data (see Section 3.2.2). Top panel: small dust grain emission model where the amorphous and crystalline silicates have a size of $0.1 \mu \mathrm{m}$. This model has a total mass of solid-state emitting grains of $\approx 4 \times 10^{19} \mathrm{~g}$, or the mass of the asteroid Ida. While this model is a reasonable match to the data, we caution that the various wiggles seen in the spectrum could instead be related to its low signal-to-noise ratio; e.g., such wiggles could be from low-level, low-frequency noise in the detector electronics. Bottom panel: large dust grain emission model where the amorphous and crystalline silicates have a size of $4.0 \mu \mathrm{m}$. The muting effect of the larger grain sizes on the solid-state emission features is evident. For grain sizes $>5 \mu \mathrm{m}$ no clear peaks from the solid-state transitions are present and it is difficult to distinguish them from the continuum (see discussion in Section 3.2.2). This model has a total mass of solid-state emitting grains that is a few times the mass of Ida.

Figure 2 of Chen et al. 2006; Figure 3 of Lisse et al. 2008), we do not explore those grain species and cannot constrain their presence. Figure 5 shows example dust emission models that are consistent with the COMICS data. There are a wide range of models that could be consistent with the data, but all such models have a total solid-state emitting dust grain mass $\lesssim 10^{20} \mathrm{~g}$ (a couple times the mass of the asteroid Ida). In comparison, other extremely dusty main-sequence stars $(\tau \gtrsim 1 \%)$ have been found to have masses for their solid-state emitting grains in the range of $10^{21}-10^{23} \mathrm{~g}$ (e.g.,
Chen et al. 2006; Lisse et al. 2008, 2009). Systems like HD 69830 ( $\tau \sim 0.01 \%$ and whose mid-infrared solid-state emission features could hide in the V488 Per COMICS spectrum) have solid-state emitting grain dust masses that encompass the limits obtained for the models shown in Figure 5, $10^{19}-10^{21} \mathrm{~g}$ (e.g., Beichman et al. 2005; Chen et al. 2006; Lisse et al. 2007, 2012).

If V488 Per is like other extremely dusty main-sequence stars and hosts a small grain population with mass of $\gtrsim 10^{21} \mathrm{~g}$, then these small grains must not produce strong solid-state emission features in the $8-13 \mu \mathrm{m}$ range. This limits the contribution from typically seen silicates and silica to $<10^{20} \mathrm{~g}$ and requires an order of magnitude more mass of dust from species like amorphous carbon and metallic iron. Given that inner planetary systems (including our own) are found to be deficient in carbon (e.g., Jura 2006, and references therein), we give preference to a scenario where these dust grains are predominantly metallic iron.

\section{Conclusions}

We present an investigation into the overall architecture of the V488 Per planetary system; Figure 2 shows companion sensitivity limits, while Figures 4 and 5 summarize dust properties.

Blackbody fits to infrared excess measurements indicate two dust populations having temperatures of 800 and $130 \mathrm{~K}$, or orbital separations of roughly 0.07 and 2.7 au, respectively. To constrain the true extent of these dust populations within the V488 Per planetary system will require additional data. Longer wavelength observations (e.g., with the Atacama Large Millimeter/submillimeter Array) can determine the outermost extent of the cool dust population, while optical/infrared interferometric observations might be capable of assessing the presence of very close-in dust.

Assuming a separated two-dust-belt interpretation is appropriate, we limit any companions within the gap between the belts to have mass $\lesssim 10 M_{\text {Jup }}$. This limit could be improved with precision radial velocity monitoring over a few year duration. Stellar-mass companions are effectively ruled out for most separations $<800$ au from V488 Per. Deeper high spatial resolution imaging (PSF FWHM $\lesssim 50$ mas) or sparse-aperture masking interferometry could push to substellar mass limits in this region.

The lack of any obvious solid-state emission features in the COMICS mid-infrared spectrum could be suggestive of dust particles composed primarily of metallic iron. If true, this might indicate the erosive bombardment and subsequent grinding down of ejecta from something resembling the deep interior of a differentiated planet. Clement et al. (2021) discuss how such a scenario may have played out during the formation of Mercury in the solar system. It is more likely to obtain such a scenario in exoplanetary systems with inner planetary systems tightly packed with Super-Earth/Earth-type planets (e.g., Pu \& Wu 2015; Izidoro et al. 2019; Clement et al. 2021, and references therein), and as such it is not unreasonable to suggest (based on the available data) that V488 Per could potentially be in the process of forming a Mercury-like planet. Planned James Webb Space Telescope/MidInfrared Instrument guaranteed time observations of V488 Per will be valuable in assessing the presence and variability of any solidstate emission features and thus further exploring this hypothesis.

C.M. acknowledges support from NASA ADAP grant No. 18-ADAP18-0233. B.K. acknowledges support from the APS 
M. Hildred Blewett Fellowship. A.W.H. acknowledges NSF grant No. AST-1517655. We thank the anonymous referee for useful comments that helped improve this paper. The authors wish to thank Bradford Holden for assistance in scheduling the APF observations presented in this paper. We thank George Rieke for useful discussion. Research at the Lick Observatory is partially supported by a generous gift from Google. Some of the data presented herein were obtained at the W.M. Keck Observatory, which is operated as a scientific partnership among the California Institute of Technology, the University of California, and the National Aeronautics and Space Administration. The Observatory was made possible by the generous financial support of the W.M. Keck Foundation. The authors wish to recognize and acknowledge the very significant cultural role and reverence that the summit of Maunakea has always had within the indigenous Hawaiian community. We are most fortunate to have the opportunity to conduct observations from this mountain. Similarly, we acknowledge that the Lick Observatory resides on land traditionally inhabited by the Muwekma Ohlone Tribe of Native Americans. This paper includes data collected by the TESS mission, which are publicly available from MAST. Funding for the TESS mission is provided by NASA's Science Mission directorate. This work is based in part on observations made with Herschel, an ESA space observatory with science instruments provided by European-led Principal Investigator consortia and with important participation from NASA. Support for Herschel work was provided by NASA through an award issued by JPL/Caltech. This research has made use of NASA's Astrophysics Data System, the SIMBAD database, and the VizieR service.

Facilities: Subaru(COMICS), APF(Levy), Shane(Hamilton), Keck(NIRC2), Herschel(PACS), TESS.

\section{ORCID iDs}

Swetha Sankar (1) https://orcid.org/0000-0002-4419-8325 Carl Melis (ib https://orcid.org/0000-0001-9834-7579 Beth L. Klein (1D https://orcid.org/0000-0001-5854-675X B. J. Fulton (1) https://orcid.org/0000-0003-3504-5316 B. Zuckerman (iD https://orcid.org/0000-0001-6809-3045 Inseok Song (D) https://orcid.org/0000-0002-5815-7372 Andrew W. Howard (10 https://orcid.org/0000-0001-8638-0320

\section{References}

Absil, O., Marion, L., Ertel, S., et al. 2021, A\&A, 651, A45 Allain, S., Fernandez, M., Martin, E. L., \& Bouvier, J. 1996, A\&A, 314, 173 Artymowicz, P. 1988, ApJL, 335, L79

Backman, D., Marengo, M., Stapelfeldt, K., et al. 2009, ApJ, 690, 1522 Ballering, N. P., Rieke, G. H., \& Gáspár, A. 2014, ApJ, 793, 57 Beichman, C. A., Bryden, G., Gautier, T. N., et al. 2005, ApJ, 626, 1061 Bonsor, A., Raymond, S. N., \& Augereau, J.-C. 2013, MNRAS, 433, 2938 Bonsor, A., Raymond, S. N., Augereau, J.-C., \& Ormel, C. W. 2014, MNRAS, 441, 2380

Chabrier, G., Baraffe, I., Allard, F., \& Hauschildt, P. 2000, ApJ, 542, 464

Chen, C. H., Sargent, B. A., Bohac, C., et al. 2006, ApJS, 166, 351

Clement, M. S., Chambers, J. E., \& Jackson, A. P. 2021, AJ, 161, 240

Currie, T., Lisse, C. M., Sicilia-Aguilar, A., Rieke, G. H., \& Su, K. Y. L. 2011, ApJ, 734, 115

Cutri, R. M. 2012, yCat, 2311, 0

Fujiwara, H., Onaka, T., Takita, S., et al. 2012b, ApJL, 759, L18

Fujiwara, H., Onaka, T., Yamashita, T., et al. 2012a, ApJL, 749, L29

Fujiwara, H., Yamashita, T., Ishihara, D., et al. 2009, ApJL, 695, L88

Gaia Collaboration, Brown, A. G. A., Vallenari, A., et al. 2021, A\&A, 649, A1

Gaidos, E., Jacobs, T., LaCourse, D., et al. 2019, MNRAS, 488, 4465

Gomes, R., Levison, H. F., Tsiganis, K., \& Morbidelli, A. 2005, Natur, 435,466
Heinze, A. N., Tonry, J. L., Denneau, L., et al. 2018, AJ, 156, 241

Henden, A. A., Levine, S., Terrell, D., \& Welch, D. L. 2015, AAS Meeting, $225,336.16$

Herschel Point Source Catalogue Working Group, Marton, G., Calzoletti, L., et al. 2020, yCat, 8106, 0

Honda, M., Kataza, H., Okamota, Y. K., et al. 2004, ApJL, 610, L49

Huang, C. X., Vanderburg, A., Pál, A., et al. 2020a, RNAAS, 4, 204

Huang, C. X., Vanderburg, A., Pál, A., et al. 2020b, RNAAS, 4, 206

Izidoro, A., Bitsch, B., Raymond, S. N., et al. 2019, A\&A, 650, A152

Johnson, B. C., Lisse, C. M., Chen, C. H., et al. 2012, ApJ, 761, 45

Jura, M. 2006, ApJ, 653, 613

Kataza, H., Okamoto, Y., Takubo, S., et al. 2000, Proc. SPIE, 4008, 1144

Kennedy, G. M., \& Wyatt, M. C. 2014, MNRAS, 444, 3164

Leggett, S. 1992, ApJS, 82, 351

Lisse, C., Chen, C., Wyatt, M., \& Morlok, A. 2008, ApJ, 673, 1106

Lisse, C. M., Beichman, C. A., Bryden, G., \& Wyatt, M. C. 2007, ApJ, 658, 584

Lisse, C. M., Chen, C. H., Wyatt, M. C., et al. 2009, ApJ, 701, 2019

Lisse, C. M., Chen, C. H., Wyatt, M. C., \& Morlok, A. 2008, ApJ, 673, 1106

Lisse, C. M., Meng, H. Y. A., Sitko, M. L., et al. 2020, ApJ, 894, 116

Lisse, C. M., Sitko, M. L., Russell, R. W., et al. 2017, ApJL, 840, L20

Lisse, C. M., Wyatt, M. C., Chen, C. H., et al. 2012, ApJ, 747, 93

Melis, C. 2016, in IAU Symp. 314, Young Stars and Planets Near the Sun, ed. J. H. Kastner, B. Stelzer, \& S. A. Metchev (Cambridge: Cambridge Univ. Press), 241

Melis, C., Olofsson, J., Song, I., et al. 2021, arXiv:2104.06448

Melis, C., Zuckerman, B., Rhee, J. H., et al. 2013, ApJ, 778, 12

Melis, C., Zuckerman, B., Rhee, J. H., \& Song, I. 2010, ApJL, 717, L57

Meng, H. Y. A., Su, K. Y. L., Rieke, G. H., et al. 2014, Sci, 345, 1032

Mermilliod, J.-C., Queloz, D., \& Mayor, M. 2008, A\&A, 488, 409

Min, M., Waters, L. B. F. M., de Koter, A., et al. 2007, A\&A, 462, 667

Mittal, T., Chen, C. H., Jang-Condell, H., et al. 2015, ApJ, 798, 87

Moór, A., Ábrahám, P., Szabó, G., et al. 2021, ApJ, 910, 27

Moór, A., Apai, D., Pascucci, I., et al. 2009, ApJL, 700, L25

Morales, F. Y., Werner, M. W., Bryden, G., et al. 2009, ApJ, 699, 1067

Morlok, A., Mason, A. B., Anand, M., et al. 2014, Icar, 239, 1

Nesvold, E. R., Naoz, S., Vican, L., \& Farr, W. M. 2016, ApJ, 826, 19

Nidever, D. L., Marcy, G. W., Butler, R. P., Fischer, D. A., \& Vogt, S. S. 2002, ApJS, 141, 503

Okamoto, Y. K., Kataza, H., Yamashita, T., et al. 2003, Proc. SPIE, 4841, 169

Olofsson, J., Juhász, A., Henning, T., et al. 2012, A\&A, 542, A90

Pakhomov, Y. V., \& Zhao, G. 2013, AJ, 146, 97

Patience, J., Ghez, A. M., Reid, I. N., \& Matthews, K. 2002, AJ, 123, 1570

Payne, M. J., Ford, E. B., Wyatt, M. C., \& Booth, M. 2009, MNRAS, 393, 1219

Pilbratt, G. L., Riedinger, J. R., Passvogel, T., et al. 2010, A\&A, 518, L1

Poglitsch, A., Waelkens, C., Geis, N., et al. 2010, A\&A, 518, L2

Price-Whelan, A. M., Hogg, D. W., Foreman-Mackey, D., \& Rix, H.-W. 2017, ApJ, 837, 20

Pu, B., \& Wu, Y. 2015, ApJ, 807, 44

Rhee, J. H., Song, I., \& Zuckerman, B. 2007, ApJ, 671, 616

Rhee, J. H., Song, I., \& Zuckerman, B. 2008, ApJ, 675, 777

Skrutskie, M. F., Cutri, R. M., Stiening, R., et al. 2006, AJ, 131, 1163

Soderblom, D. R., Hillenbrand, L. A., Jeffries, R. D., Mamajek, E. E., \& Naylor, T. 2014, in Protostars and Planets VI, ed. H. Beuther et al. (Tuscon: Univ. Arizona), 219

Song, I., Zuckerman, B., Weinberger, A. J., \& Becklin, E. E. 2005, Natur, 436, 363

Stassun, K. G., Oelkers, R. J., Paegert, M., et al. 2019, AJ, 158, 138

Stauffer, J., Hartmann, L., Burnham, J., \& Jones, B. 1985, ApJ, 289, 247

Strassmeier, K. G., Fekel, F. C., Bopp, B. W., Dempsey, R. C., \& Henry, G. W. 1990, ApJS, 72, 191

Su, K. Y. L., Rieke, G. H., Melis, C., et al. 2020, ApJ, 898, 21

Su, K. Y. L., Rieke, G. H., Stapelfeldt, K. R., et al. 2009, ApJ, 705, 314

Tajiri, T., Kawahara, H., Aizawa, M., et al. 2020, ApJS, 251, 18

Vican, L., Schneider, A., Bryden, G., et al. 2016, ApJ, 833, 263

Vogt, S. S. 1987, PASP, 99, 1214

Vogt, S. S., Radovan, M., Kibrick, R., et al. 2014, PASP, 126, 359

Wizinowich, P. 2013, PASP, 125, 798

Wizinowich, P., Acton, D. S., Shelton, C., et al. 2000, PASP, 112, 315

Wright, N. J., Drake, J. J., Mamajek, E. E., \& Henry, G. W. 2011, ApJ, 743, 48

Yelda, S., Lu, J. R., Ghez, A. M., et al. 2010, ApJ, 725, 331

Zuckerman, B. 2015, ApJ, 798, 86

Zuckerman, B., Fekel, F. C., Williamson, M. H., Henry, G. W., \& Muno, M. P. 2008, ApJ, 688, 1345

Zuckerman, B., Melis, C., Rhee, J. H., Schneider, A., \& Song, I. 2012, ApJ, 752,58 